\title{
INTEGRABILITY OF NONNEGATIVE TRIGONOMETRIC SERIES
}

\author{
RALPH PHILIP BOAS, JR.*
}

(Received June 20,1962)

1. Introduction. It is known that if a trigonometric series converges everywhere to a nonnegative sum $f(x)$ then $f$ is integrable and the series is a Fourier series [5, p.328], whereas when a trigonometric series converges to a nonnegative sum only in an interval $(a, b)$, its sum is integrable over interior intervals, but is integrable over $(a, b)$ if and only if the integrated series converges at the endpoints of the interval [5, p.372, no.14]. However, the sum belongs to $L^{1-\delta}$, for every positive $\delta$, over the whole interval $(a, b)[5, \mathrm{p} .371$, no.13]. I shall give a simple proof of this last result by showing first that $(x-a)^{\alpha}(b-x)^{\alpha} f(x)$ is integrable over $(a, b)$ for every positive $\alpha$.

There is another natural sense in which a nonnegative function $f$ can be associated with a trigonometric series, namely that the coefficients in the series are the Fourier coefficients of $f$ in a generalized sense. If we consider the case when $f$ is integrable except in the neighborhood of one point, which we may take to be 0 , we can obtain necessary and sufficient conditions for the integrability of $x^{\alpha} f(x)$ for certain nonnegative values of $\alpha$. These may be considered as analogues of the known results that connect integrability of $x^{\alpha} f(x)$ with the convergence of $\Sigma c_{n} n^{-\alpha-1}$ when $\alpha<0$ (see, for example, [1], [2], [4], where further references are given).

\section{Convergent trigonometric series.}

THEOREM 1. If a trigonometric series $\Sigma c_{n} \mathrm{e}^{i n x}$ converges in some $(0, \delta)$ to sum $f(x)$ and $f(x) \geqq 0$ in this neighborhood then $x^{\alpha} f(x) \in L$ in a right-hand neighborhood of 0 for every positive $\alpha$.

Proof. Since the series converges in an interval, $c_{n} \rightarrow 0$. We know that $f$ is integrable on every $(a, b), 0<a<b<\delta$. (Cf. [5], pp.328 and 371, no.13.) Since the Fourier series of the function equal to $f(x)$ on $(a, b)$ and to 0 elsewhere is equiconvergent with $\Sigma c_{n} \mathrm{e}^{i n x}$ over any closed subinterval of $(a, b)$ ([5], p.330), we can integrate $\Sigma c_{n} \mathrm{e}^{i n x}$ formally over $(x, \varepsilon)$, where $0<x<\varepsilon<\delta$, and obtain an integral of $f$. Since the series $\Sigma c_{n} \mathrm{e}^{i n x} /(\mathrm{in})$ is a Fourier series, and indeed the Fourier series of a function that belongs to every $L^{p}(p<\infty)$, by the HausdorffYoung theorem, $\int_{x}^{\varepsilon} f(t) d t \in L^{p}$ for every $p$. Then by Hölder's inequality

\footnotetext{
* President's Fellow, Northwestern University.
} 


$$
\begin{aligned}
\alpha^{-1} \int_{0}^{\varepsilon} t^{\alpha} f(t) d t & =\int_{0}^{\varepsilon} f(t) d t \int_{0}^{t} x^{\alpha-1} d x=\int_{0}^{\varepsilon} x^{\alpha-1} d x \int_{x}^{\varepsilon} f(t) d t \\
& \leqq\left\{\int_{0}^{\varepsilon} d x\left[\int_{x}^{\varepsilon} f(t) d t\right]^{p}\right\}^{1 / p}\left\{\int_{0}^{\varepsilon} x^{\left(\alpha-1 ; p^{\prime}\right.} d x\right\}^{1 / p^{\prime}}<\infty
\end{aligned}
$$

provided that $p^{\prime}=p /(p-1)>1 / \alpha$.

THEOREM 2. With the hypotheses of Theorem $1, f \in L^{1-\eta}$ in a right-hand neighborhood of 0 , for every positive $\eta$.

We have

$$
\begin{aligned}
\int_{0}^{\Sigma} f(t)^{1-\eta} d t & =\int_{0}^{\varepsilon} f(t)^{1-\eta} t^{\lambda} t^{-\lambda} d t \\
& \leqq\left\{\int_{0}^{\varepsilon} f(t) t^{\lambda /(1-\eta)} d t\right\}^{1-\eta}\left\{\int_{0}^{\varepsilon} t^{-\lambda^{\prime} \eta} d t\right\}^{\eta}<\infty
\end{aligned}
$$

by Hölder's inequality, provided that $0<\lambda<\eta$.

3. Generalized sine series. We now consider generalized Fourier series of nonnegative functions. We discuss sine and cosine series separately.

THEOREM 3. If $0<\alpha<1, x^{\alpha} f(x) \in L$, and

$$
b_{n}=\frac{2}{\pi} \int_{0}^{\pi} f(x) \sin n x d x
$$

then

$$
\sum n^{-\alpha-1} b_{n}
$$

converges.

This is a well-known elementary fact for $\alpha=0$. For $\alpha=1$, see Theorem 6 .

We have

$$
\begin{aligned}
\frac{1}{2} \pi \sum_{m}^{M} n^{-\alpha-1} b_{n} & =\sum_{m}^{M} n^{-\alpha-1} \int_{0}^{\pi} f(x) \sin n x d x \\
& =\int_{0}^{\pi} f(x) \sum_{m}^{M} n^{-\alpha-1} \sin n x d x=\int_{0}^{\varepsilon}+\int_{\varepsilon}^{\pi}=I_{1}+I_{2} .
\end{aligned}
$$

In $I_{1}$, we have

$$
\begin{aligned}
\left|\sum_{m}^{M} \frac{\sin n x}{n^{\alpha+1}}\right| & \leqq \sum_{m}^{[1 / x]}\left|\frac{\sin n x}{n x} \frac{x}{n^{\alpha}}\right|+\sum_{[1 ; x\}}^{M}\left|\frac{\sin n x}{n^{\alpha+1}}\right| \\
& \leqq x \sum_{m}^{[1 ; x]} n^{-\alpha}+\sum_{[1, x]}^{M} n^{-\alpha-1} \leqq A x^{\alpha}
\end{aligned}
$$


where $A$ is independent of $m$ and $M$. Hence

$$
\left|I_{1}\right| \leqq A \int_{0}^{\varepsilon}|f(x)| x^{\alpha} d x
$$

In $I_{2}$, we have

$$
\left|\sum_{m}^{M} n^{-\alpha-1} \sin n x\right| \leqq \sum_{m}^{M} n^{-\alpha-1} \leqq A m^{-\alpha}
$$

We obtain

$$
\left|I_{2}\right| \leqq A m^{-\alpha} \int_{\varepsilon}^{\pi}|f(x)| d x
$$

If we take $\varepsilon$ small and then $m$ large we can therefore make $I_{1}+I_{2}$ arbitrarily small, and so (2) converges.

Theorem 3 assumes nothing about the sign of $f(x)$. When $f(x) \geqq 0$, it has a converse.

THEOREM 4. If $0<\alpha<1, f(x) \geqq 0$ on $(0, \pi), x f(x) \in L, b_{n}$ are defined by (1), and $\Sigma n^{-\alpha-1} b_{n}$ converges, then $f(x) x^{\alpha} \in L$.

We have

$$
\begin{aligned}
\frac{1}{2} \pi \sum_{1}^{M} n^{-\alpha-1} b_{n} & =\sum_{1}^{M} n^{-\alpha-1} \int_{0}^{\pi} f(x) \sin n x d x \\
& =\int_{0}^{\pi} f(x) \sum_{1}^{M} n^{-\alpha-1} \sin n x d x .
\end{aligned}
$$

Since $\sum n^{-1} \sin n x$ has nonnegative partial sums, partial summation shows that $\Sigma n^{-\alpha-1} \sin n x$ also has nonnegative partial sums. Hence by Fatou's lemma

$$
\int_{0}^{\pi} f(x) \sum_{1}^{\infty} n^{-\alpha-1} \sin n x d x \leqq \lim _{M \rightarrow \infty} \frac{1}{2} \pi \sum_{1}^{M} n^{-\alpha-1} b_{n} .
$$

Now

$$
\sum_{1}^{\infty} n^{-\alpha} \cos n t \sim A t^{\alpha-1}
$$

and so

$$
\sum_{1}^{\infty} n^{-\alpha-1} \sin n x=\int_{0}^{x} \sum_{1}^{\infty} n^{-\alpha} \cos n t d t \sim A x^{\alpha} \quad(x \rightarrow 0)
$$

Hence (3) implies that $\int_{0}^{\pi} f(x) x^{\alpha} d x<\infty$. We have not used the full force of 
the hypothesis that $\Sigma n^{-\alpha-1} b_{n}$ converges; it would be enough for this series to have a sequence of bounded partial sums.

Theorem 4 is still true when $\alpha=0$ but the proof is slightly different.

THEOREM 5. If $f(x) \geqq 0$ on $(0, \pi), b_{n}$ are defined by (2), and $\Sigma b_{n} / n$ converges then $f(x) \in L$.

The reasoning leading to (3) is unchanged when $\alpha=0$, and the series on the left is now equal to $(\pi-x) / 2$. Hence

$$
\int_{0}^{\pi} f(x)(\pi-x) d x \leqq \lim _{M \rightarrow \infty} \inf \pi \sum_{1}^{M} n^{-1} b_{n} .
$$

Since $x f(x) \in L,(4)$ shows that $f(x) \in L$.

When $\alpha=1$, Theorem 4 is vacuous and Theorem 3 fails; as an example we may take an odd function equal to $x^{-2}(\log x)^{-2}$ in a right-hand neighborhood of 0 . We have the following substitute.

THEOREM 6. If $x f(x) \log (1 / x) \in L$ and $b_{n}$ is defined by (1) then $\Sigma n^{-2} b_{n}$ converges; if $\Sigma n^{-2} b_{n}$ converges and $f(x) \geqq 0$ then $x f(x) \log (1 / x) \in L$.

If $x f(x) \log (1 / x) \in L$ we have

$$
\begin{aligned}
\frac{1}{2} \pi \sum_{m}^{M} n^{-2} b_{n} & =\sum_{m}^{M} n^{-2} \int_{0}^{\pi} f(x) \sin n x d x \\
& =\int_{0}^{\pi} f(x) \sum_{m}^{M} n^{-2} \sin n x d x=\int_{0}^{\varepsilon}+\int_{\varepsilon}^{\pi}=I_{1}+I_{2} .
\end{aligned}
$$

By the same reasoning as in Theorem 3 we see that $\sum_{m}^{M} n^{-2} \sin n x$ is $O(x \log (1 / x))$ uniformly in $m$ and $M$ as $x \rightarrow 0$, and $O(1 / m)$ for $x>\varepsilon$ as $m \rightarrow \infty$. The conclusion follows.

Conversely,

$$
\frac{1}{2} \pi \sum_{1}^{M} n^{-2} b_{n}=\int_{0}^{\pi} f(x) \sum_{1}^{M} n^{-2} \sin n x d x,
$$

and if $\Sigma n^{-2} b_{n}$ converges we have

$$
\int_{0}^{\pi} f(x) \sum_{1}^{\infty} n^{-2} \sin n x d x \leqq \frac{1}{2} \pi \sum_{1}^{\infty} n^{-2} b_{n} .
$$

Now $\sum_{1}^{\infty} n^{-1} \cos n x=-\log (2 \sin x / 2)$ and hence $\sum_{1}^{\infty} n^{-2} \sin n x \sim x \log (1 / x)$ as $x \rightarrow 0$. The conclusion follows.

4. Generalized cosine series. For cosine series the situation is somewhat 
different. If we assume $f(x) \geqq 0$ then the existence of the cosine coefficient $a_{0}$ automatically makes $f \in L$. We shall therefore suppose that $a_{0}=0$, and require $f(x) \geqq 0$ only in a neighborhood of 0 . We can then work with a wider range of $\alpha$ than in $\S 3$.

THEOREM 7. If $0<\alpha<2, x^{\alpha} f(x) \in L$, and

$$
a_{n}=\frac{2}{\pi} \int_{\rightarrow 0}^{\pi} f(x) \cos n x d x, \quad n=1,2, \ldots
$$

then

$$
\Sigma n^{-\alpha-1} a_{n}
$$

converges.

We have

$$
\frac{1}{2} \pi \sum_{m}^{M} n^{-\alpha-1} a_{n}=\int_{0}^{\pi} f(x) \sum_{m}^{M} \frac{\cos n x}{n^{\alpha+1}} d x=-\int_{0}^{\pi} f(x) \sum_{m}^{M} \frac{1-\cos n x}{n^{\alpha+1}} d x
$$

since $\int_{0}^{\pi} f(x) d x=0$. Thus

$$
\frac{1}{2} \pi \sum_{m}^{M} n^{-\alpha-1} a_{n}=-\left(\int_{0}^{\varepsilon}+\int_{\varepsilon}^{\pi}\right) f(x) \sum_{m}^{M} \frac{2 \sin ^{2} n x / 2}{n^{\alpha+1}} d x=I_{1}+I_{2} .
$$

In $I_{1}$ we have

$$
\sum_{m}^{M} \frac{\sin ^{2} n x / 2}{n^{\alpha+1}} \leqq \sum_{m}^{[1 / x]}+\sum_{[1, x]}^{M} \leqq \frac{1}{4} x^{2} \sum_{m}^{[1, x\rfloor} n^{1-\alpha}+\sum_{\lfloor 1 / x]}^{M} n^{-\alpha-1}<A x^{\alpha} .
$$

In $I_{2}$,

$$
\sum_{m}^{M} n^{-\alpha-1} \sin ^{2} n x / 2 \leqq A m^{-\alpha}
$$

as in the proof of Theorem 3. The convergence of (6) then follows.

THEOREM 8. If $0<\alpha<2, f \in L$ in every $(\varepsilon, \pi), \varepsilon>0, f(x) \geqq 0$ in a right-hand neighborhood of $0, a_{n}$ are defined by (5) with $a_{0}=0$, and $\Sigma n^{-\alpha-1} a_{n}$ converges, then $f(x) x^{\alpha} \in L$.

Thus $\alpha=1$ is not an exceptional case for cosine series.

We have

$$
\frac{1}{2} \pi \sum_{1}^{M} n^{-\alpha-1} a_{n}=\int_{0}^{\pi} f(x) \sum_{1}^{M} n^{-\alpha-1} \cos n x d x .
$$


Now $\sum_{1}^{\infty} n^{-\beta} \cos n x$ has its partial sums uniformly bounded below for $\beta$ sufficiently near 1 ; hence, by partial summation, so does $\Sigma n^{-\alpha-1} \cos n x, 0<\alpha<1$. Let $-K$ be a lower bound for the partial sums of the latter series; since $a_{0}=0$, we have

$$
\frac{1}{2} \pi \sum_{1}^{M} n^{-\alpha-1} a_{n}=\int_{0}^{\pi} f(x)\left\{\sum_{1}^{M} n^{-\alpha-1} \cos n x+K\right\} d x .
$$

As in Theorem 4, it now follows by Fatou's lemma that

$$
\int_{0}^{\pi} f(x)\left\{\sum_{1}^{\infty} n^{-\alpha-1} \cos n x+K\right\} d x
$$

converges, and (again since $a_{0}=0$ ) therefore so does

$$
\int_{0}^{\pi} f(x) \sum_{1}^{\infty} n^{-\alpha-1}(1-\cos n x) d x
$$

But

$$
\sum_{1}^{\infty} n^{-\alpha-1}(1-\cos n x)=\int_{0}^{x} \sum_{1}^{\infty} n^{-\alpha} \sin n x d x \sim A x^{\alpha}(x \rightarrow 0)
$$

([5], p. 186), and so the conclusion follows.

In the case $\alpha=0$, conditions for the convergence of (6), i.e. of $\Sigma n^{-1} a_{n}$, are known (cf. [5]. p.228, no.8; [3], p.96).

\section{REFERENCES}

[1] CHEN YUNG-Ming, Some further asymptotic properties of Fourier constants, Math. Zeit. 69(1958), 105-120.

[2] J. M. GonZALEZ-FERnÁndEZ, Integrability of trigonometric series, Proc. Amer. Math. Soc. 9(1958), 315-319.

[3] G. H. HARDY AND W. W. Rogosinski, Fourier series, Cambridge, 1944.

[4] S. O'SHEA, Note on an integrability theorem for sine series, Quart. Journ. Math. Oxford (2), 8(1957), 279-281.

[5] A.ZYGmund, Trigonometric series, 2d ed., vol. I, Cambridge 1959.

NORTHWESTERN UNIVERSITY

EVANSTON ILLINOIS 